\title{
Pharmacologically Induced Sex Hormone Fluctuation Effects on Resting-State Functional Connectivity in a Risk Model for Depression: A Randomized Trial
}

\author{
Patrick MacDonald Fisher ${ }^{1,2}$, Camilla Borgsted Larsen ${ }^{1,2}$, Vincent Beliveau ${ }^{1,2,3}$, Susanne Henningsson ${ }^{1,4}$, \\ Anja Pinborg ${ }^{3,5}$, Klaus Kähler Holst ${ }^{6}$, Peter Steen Jensen ${ }^{1,2}$, Claus Svarer ${ }^{1,2}$, Hartwig Roman Siebner ${ }^{1,3,4,7}$, \\ Gitte Moos Knudsen ${ }^{1,2,3}$ and Vibe Gedsoe Frokjaer*, 1,2 \\ 'Center for Integrated Molecular Brain Imaging, Copenhagen, Denmark; ${ }^{2}$ Neurobiology Research Unit, Copenhagen University Hospital \\ Rigshospitalet, Copenhagen, Denmark; ${ }^{3}$ Faculty of Health and Medical Sciences, University of Copenhagen, Copenhagen, Denmark; ${ }^{4}$ Danish \\ Research Centre for Magnetic Resonance, Centre for Functional and Diagnostic Imaging and Research, Hvidovre Hospital, Hvidovre, Denmark; \\ ${ }^{5}$ The Fertility Clinic, Copenhagen University Hospital Rigshospitalet, Copenhagen, Denmark; ${ }^{6}$ Department of Biostatistics, University of \\ Copenhagen, Copenhagen, Denmark; ${ }^{7}$ Department of Neurology, Bispebjerg Hospital, Copenhagen, Denmark
}

\begin{abstract}
Women are at relatively greater lifetime risk for depression than men. This elevated risk in women is partly due to heightened risk during time periods characterized by marked fluctuations in sex hormones, including postpartum and perimenopausal periods. How sex hormone fluctuations contribute to heightened risk is not fully understood but may involve intrinsic functional connectivity. We induced a biphasic ovarian sex hormone fluctuation using the gonadotropin-releasing hormone agonist ( $\mathrm{GnRHa}$ ) goserelin to determine, with a randomized placebo-controlled design, intervention effects on or GnRHa-provoked depressive symptoms associations with change in resting-state functional connectivity (rs-FC) in 58 healthy women for six seeds (amygdala, hippocampus, anterior cingulate cortex, dorsal raphe, median raphe, and posterior cingulate cortex). GnRHa intervention did not significantly affect rs-FC in any seeds. Considering the GnRHa group only, the emergence of depressive symptoms following intervention was positively associated with amygdala-right temporal cortex and negatively associated with hippocampus-cingulate rs-FC. A test for mediation suggested that rs-FC changes in these networks marginally mediated the association between decrease in estradiol and increase in depressive symptoms in the GnRHa group ( $p=0.07)$. Our findings provide novel evidence-linking changes in rs-FC networks, the emergence of depressive symptoms and sex hormone fluctuations. Notably, we observed evidence that changes in rs-FC may represent a key neurobiological intermediary between molecular changes induced by hormone fluctuations and the emergence of depressive symptoms. Taken together, our findings indicate that sex hormone fluctuations may contribute to heightened risk for developing depressive symptoms by affecting intrinsic functional connectivity of key limbic brain structures. Neuropsychopharmacology (2017) 42, 446-453; doi:I 0.1038/npp.2016.208; published online 19 October 2016
\end{abstract}

\section{INTRODUCTION}

Lifetime risk for major depression is twice as high in women than in men (Kessler et al, 1993). Periods of ovarian sexsteroid hormone fluctuation may contribute to this increased risk. Postpartum depression (Munk-Olsen et al, 2006), premenstrual dysphoric disorder, and major depression with onset during menopausal transition (Freeman et al, 2006, 2013) are mood disorders coincident with fluctuations, or a rapid decline, in ovarian sex-steroid hormones. Recent evidence support that only a certain subgroup of women is particularly sensitive to changes in sex steroid hormone milieu (Mehta et al, 2014). Nevertheless, owing to a

* Correspondence: Dr VG Frokjaer, Neurobiology Research Unit 6931, Copenhagen University Hospital, Rigshospitalet, Blegdamsvej 9, Copenhagen DK-2 I00, Denmark, Tel: +45 354567I2, Fax: +45 354567I3,

E-mail: vibe@nru.dk

Received 21 April 2016; revised 26 July 2016; accepted II September 2016; accepted article preview online 2I September 2016 prevalence of $13 \%$ of postpartum depression (MeltzerBrody et al, 2013) and $10-30 \%$ of depressive episodes in menopausal transition even in women with no previous history of depression (Freeman et al, 2013) these disorders constitute a major public health problem. Longitudinal data show that fluctuation in estradiol is the strongest risk factor for developing subclinical/manifest depressive episodes during menopausal transition (Freeman et al, 2006). That excess risk attenuates from the time menopausal state is established (Freeman et al, 2013). In post-partum depression (PPD), estradiol sensitivity is a core risk mechanism (Bloch et al, 2000; Guintivano et al, 2014). Yet, delineating possible risk mechanisms associated with sensitivity to sex-steroid hormone fluctuations requires further investigation.

Several limbic brain regions are sensitive to ovarian sex steroid hormones. Specifically, the amygdala, hypothalamus, hippocampus, and brainstem highly express estradiol and progesterone hormone receptors, therefore integrating 
steroid hormone information (Barth et al, 2015). Ovarian steroid hormones can also affect serotonin neurotransmission derived from the dorsal and median raphe, which in turn can modulate depression-related features of brain function (Bethea et al, 2002). Therefore, ovarian steroid hormone fluctuations may potently affect brain function in areas critically associated with depressive disorders including their functional connectivity. Both clinical and experimental evidence supports that estrogen affects brain structure (Gould et al, 2000; Yankova et al, 2001) and function (Comasco et al, 2014; Sellers et al, 2015). Estrogen effects in the hippocampus can occur rapidly over the rodent estrous cycle, detectable within $24 \mathrm{~h}$ (Woolley and McEwen, 1992) and over the menstrual cycle in humans (Protopopescu et al, 2008). Initial human studies support a possible temporary neuroprotective effect of hormonal replacement in early menopause as reflected by increased hippocampal volume (Lord et al, 2008), which seemingly maintains cognitive function including verbal memory in the menopausal transition phase (Amin et al, 2006) independent of longterm cognitive outcomes (Lethaby et al, 2008). A recent single-subject resting-state functional connectivity (rs-FC) study observed a progesterone (luteal-phase driven) associated increase in rs-FC between hippocampus and bilateral dorsolateral prefrontal cortex and sensorimotor cortex (Arelin et $a l, 2015)$. A larger study demonstrated that default-mode network (DMN) and executive control network resting-state dynamics were altered by menstrual cycle and oral contraception (Petersen et al, 2014). However, a third study observed no such effects of menstrual cycle (Hjelmervik et al, 2014). Critically, these studies did not include measurement of serum estradiol, leaving unclear the association between changes in ovarian steroid hormone levels, rs-FC, and related behavioral changes.

Clinical evidence supports disturbances in task- and nontask-oriented brain function/connectivity in PPD relative to healthy postpartum women with fMRI. Amygdala response to threat-related emotional cues is blunted in PPD relative to healthy postpartum women (Moses-Kolko et al, 2010; Silverman et al, 2011). Further, resting-state fMRI studies have shown that patients with PPD have attenuated amygdala rs-FC to DMN hubs (Chase et al, 2013) and disrupted rs-FC in several regions known to be affected in depression (eg, anterior cingulate, amygdala, hippocampus, and dorsolateral prefrontal cortex) (Deligiannidis et al, 2013).

We have recently shown that sex-steroid hormone fluctuations elicited by the gonadotropin-releasing hormone agonist (GnRHa) goserelin (Frokjaer et al, 2015) was associated with alterations in reactivity to emotionally salient faces (Henningsson et al, 2015) and processing rewarding stimuli (Macoveanu et al, 2016). However, the effects of GnRHa manipulation on resting-state connectivity in brain networks implicated in sex hormone related mental illness has not been evaluated. Goserelin initially stimulates and subsequently downregulates ovarian hormone production, in particular estradiol, by desensitising pituitary gland $\mathrm{GnRH}$ receptors. Importantly, GnRHa intervention provoked depressive symptoms in some participants, as detailed in Frokjaer et al (2015) making it possible to study neurobiological mechanisms sensitive to transient fluctuations in ovarian sex-steroids and the potential coupling of these mechanisms to the emergence of depressive symptoms.
Here we evaluated GnRHa intervention effects on seedbased rs-FC networks for six brain areas strongly associated with mood regulation and major depression: the amygdala, hippocampus, anterior cingulate cortex (ACC), posterior cingulate cortex (PCC), dorsal raphe, and median raphe. Furthermore, we evaluated the relation between change in rsFC and depressive symptoms in the GnRHa group to identify resting-state networks associated with individual variability in intervention sensitivity. Finally, building on our previous observation that the magnitude of GnRHa induced estradiol decline was associated with the emergence of depressive symptoms (Frokjaer et al, 2015), we evaluated mediation models linking observed effects of $\mathrm{GnRHa}$ intervention on change in estradiol levels, rs-FC and the emergence of depressive symptoms.

\section{MATERIALS AND METHODS}

\section{Participants, Intervention, and Assessment Timing}

Participants were recruited by internet advertisements. Inclusion criteria comprised regular menstrual cycles (duration 23-35 days), normal hormone blood tests, neurological and gynaecological examination, and no history of neurological, gynaecological, or psychiatric disorder. Demographic and clinical characteristics are detailed elsewhere (Frokjaer et al, 2015).

Sixty-three healthy women were enrolled in a block randomized, placebo-controlled and double-blind intervention study. Block randomization was performed using an algorithm to balance the distribution of 5-HTTLPR genotype status (ie, $\mathrm{L}_{\mathrm{A}} \mathrm{L}_{\mathrm{A}}$ vs non- $\mathrm{L}_{\mathrm{A}} \mathrm{L}_{\mathrm{A}}$ ) between groups as previous studies suggest that 5-HTTLPR gene variants may influence the risk of developing depressive episodes in the context of sex-steroid hormone changes (eg, pre- to postpartum transition) (Mehta et al, 2012; Sanjuan et al, 2008). All participants (mean age $24.3 \pm 4.9$ years) had regular menstrual cycles and normal blood tests including follicle stimulating hormone, thyroid stimulating hormone, and androgen status at baseline. Five participants were excluded: anovulation $(N=1)$, became pregnant $(N=1)$, withdrew for personal reasons $(N=1)$, MRI artefact $(N=1)$, and missed menstrual timing at follow-up $(N=1)$. Consequently, data from 58 participants were available for analyses (Table 1). Participants received a $3.6 \mathrm{mg}$ goserelin implant $(n=29)$ or saline injection $(n=29)$ in a natural cycle during the midluteal phase (GnRHa: cycle day $22.9 \pm 3.6$ and placebo: $22.7 \pm 2.3$ ) by a gynaecologist uninvolved in subsequent data collection or analysis. Imaging, depressive symptoms, and estradiol measures were acquired and evaluated by individuals blinded to group status except for analyses related to only the GnRHa group, for which blinding was not possible. Blinding was maintained during statistical analysis using an arbitrary binary coding for group status.

Baseline measures were placed in the midfollicular phase when ovarian hormone levels are most stable and time since the postovulatory estradiol drop is maximized. Follow-up was placed post bleeding at a time point late enough to allow the GnRHa group to enter early ovarian suppression $(17.8 \pm 2.8$ days after intervention), whereas the placebo group being concurrently in the follicular phase of the next menstrual cycle. 
Table I Demographic Information at Baseline

\begin{tabular}{lrcc}
\hline Intervention & Placebo & GnRHa & p-value \\
\hline N & \multicolumn{1}{c}{29} & 29 & 1 \\
5-HTTLPR & $10 / 19$ & $10 / 19$ & 1 \\
Age (years) & $25.4 \pm 6.0$ & $23.2 \pm 3.0$ & 0.08 \\
Body mass index & $23.4 \pm 3.9$ & $23.2 \pm 2.4$ & 0.80 \\
Neuroticism & $86.6 \pm 22.1$ & $86.5 \pm 21.0$ & 0.99 \\
HDRS-17 & $1.6 \pm 2.2$ & $1.2 \pm 1.6$ & 0.46 \\
PSS & $9.7 \pm 5.7$ & $9.4 \pm 4.6$ & 0.82 \\
PMSQ & $7.6 \pm 6.2$ & $6.6 \pm 4.7$ & 0.48 \\
Cycle length (days) & $28.8 \pm 3.8$ & $30.6 \pm 3.9$ & 0.08 \\
FSH & $5.7 \pm 2.2$ & $6.2 \pm 1.9$ & 0.36 \\
Estradiol & $0.19 \pm 0.11$ & $0.19 \pm 0.10$ & 0.97 \\
Progesterone & $1.7 \pm 0.7$ & $2.7 \pm 5.2$ & 0.35 \\
Antral follicle count & $28.4 \pm 14.4$ & $26.2 \pm 10.7$ & 0.52 \\
\hline Val & &
\end{tabular}

Values expressed as mean $\pm S D ; 5-H T T L P R$ indicates $L_{A} L_{A} / S$-carriers; neuroticism score from NEO PI-R; HDRS- 17, Hamilton Depression Rating Scale17 score; PSS, perceived stress score; PMSQ, premenstrual symptoms questionnaire score; FSH, follicle-stimulating hormone measured at cycle days $2-$ 5 during screening procedures; Antral follicle count, assessed by ultrasound examination of the ovaries. $p$-value reflects $\chi^{2}$-test or two-sample $t$-test for differences between groups.

Hamilton 17-item depression rating scale and estradiol concentrations, primary outcome measures, were acquired at Rigshospitalet within $0 \pm 2$ days from the fMRI at baseline and follow-up. Estradiol was determined in serum with a detection range of $0.04-78.9 \mathrm{nmol} / \mathrm{l}$ (Frokjaer et al, 2015). As stated previously, samples with levels below the detection range were imputed to $0.04 \mathrm{nmol} / \mathrm{l}$ (Frokjaer et al, 2015). Estradiol values were $\log _{2}$ transformed prior to analyses.

The study was registered and approved by the local ethics committee under the protocol number H-2-2010-108. Data were collected between January 2011 and December 2012. Written informed consent was obtained from all participants. Resting-state data included here was included in a previous multimodal neuroimaging methods study of raphe functional connectivity (Beliveau et al, 2015). The broader study design and intervention effects on brain serotonin transporter levels were published (Frokjaer et al, 2015).

\section{Magnetic Resonance Imaging Acquisition and Pre-Processing}

Data were acquired on a Siemens $3 \mathrm{~T}$ Verio scanner at the Danish Research Centre for Magnetic Resonance using a 32-channel head coil (Beliveau et al, 2015). We acquired high-resolution sagittal, magnetization-prepared rapid-gradient echo T1-weighted structural images (TR/TE/TI $=1900$ $/ 2.32 / 900 \mathrm{~ms}$, flip angle $=9^{\circ}$, in-plane matrix $=256 \times 256$, slices $=224$, voxel size $=0.9 \times 0.9 \times 0.9 \mathrm{~mm}$, GRAPPA acceleration factor $=2$ ) and a $10 \mathrm{~min}$ resting-state scan using a T2*-weighted gradient echo-planar imaging sequence $(280$ volumes, $\mathrm{TR} / \mathrm{TE}=2150 / 26 \mathrm{~ms}$, flip-angle $=78^{\circ}$, in-plane matrix $=64 \times 64$, slices $=42$, voxel size $=3 \times 3 \times 3 \mathrm{~mm}$, no gap, GRAPPA acceleration factor $=2$, interleaved slice acquisition). Participants were instructed to close their eyes but not to fall asleep during the resting-state scan. Data collected were part of a broader MRI scan session wherein participants completed tasks while in the scanner. Restingstate fMRI data, a primary outcome measure, were acquired after the structural scans, prior to fMRI tasks.

The dorsal and median raphe are small structures adjacent to both cerebrospinal fluid (CSF) and white-matter (WM), particularly sensitive to noise sources. Therefore, a specific pre-processing pipeline accounting for these noise sources was used as described previously (Beliveau et al, 2015). For remaining seeds, functional images were pre-processed using SPM8 (http://www.fil.ion.ucl.ac.uk/spm/software/spm8/). Functional images were realigned, co-registered with the T1-weighted structural image, normalized into Montreal Neurological Institute (MNI) space (final voxel size: $2 \times 2 \times 2 \mathrm{~mm}$ ) then smoothed with an $8 \mathrm{~mm}$ Gaussian kernel. Time-series were band-pass filtered $(0.008-0.09 \mathrm{~Hz})$ and temporal processing of resting-state data was performed in Conn v13.p (Whitfield-Gabrieli and Nieto-Castanon, 2012). Physiological and other noise sources were estimated using aCompCor (Behzadi et al, 2007). Volumes with excessive motion ( $>2 \mathrm{~mm} /$ degrees) or image variability ( $>4$ SDs) were flagged using Artifact Detection Tools (http://www. nitrc.org/projects/artifact_detect/) and censored from analyses.

\section{rs-FC Analyses}

We generated rs-FC maps for each participant for six seeds: ACC, amygdala, dorsal raphe, hippocampus, median raphe, and PCC. Bilateral amygdala and ACC seeds were defined using WFU Pickatlas (http://fmri.wfubmc.edu/software/pick atlas). The bilateral hippocampus seed was defined by $5 \mathrm{~mm}$ radius spheres $\sim(-25,-26,-10)$ and $(25,-26,-10)$ (Deligiannidis et al, 2013). We used the PCC seed detailed in Conn to delineate the $\mathrm{DMN}$, defined by a $10 \mathrm{~mm}$ radius sphere $\sim(-6,-52,40)$ (Fox et al, 2005). Subject-specific dorsal and median raphe seeds were built using an iterative method based on serotonin transporter positron emission tomography scans as described previously (Beliveau et al, 2015). Dorsal and median raphe seeds were constrained to 115 and $64 \mathrm{~mm}^{3}$, consistent with anatomical estimates (Baker et al, 1990; Kranz et al, 2012). Owing to their small volume and proximity to noise sources, a conservative motion threshold was imposed (Beliveau et al, 2015) and data from only 40 participants ( 20 goserelin and 20 placebo) were included in analyses. Rs-FC for each seed was evaluated for each participant in separate linear models.

Voxel-level rs-FC maps were generated by calculating the correlation coefficient between a seed time-series and each brain voxel. Coefficients were converted to $z$-scores using Fisher's r-to-z transformation. Single-subject rs-FC maps were entered into second-level models to determine main effects of connectivity, effects of GnRHa intervention and correlation with change in depressive symptoms. For all analyses, WM, CSF, and cerebellum voxels were excluded using masks based on WFU Pickatlas. To address the issue of multiple comparisons, 3dClustSim in AFNI (http://afni. nimh.nih.gov/afni, v16.2.01, 8 July 2016) was used to determine a cluster extent threshold unlikely to have occurred by chance, a family-wise error correction. Adjusting for the six seeds and two sets of comparisons (ie, group effect and change in depressive symptoms), we determined a 
cluster extent threshold unlikely to have occurred by chance at $\alpha<0.004$ (ie, $0.05 / 12$ ). This corresponded to a voxel-level threshold of $p<0.001$ and a whole-brain cluster extent threshold of $k>168$ voxels.

\section{Statistics}

We evaluated GnRHa intervention effects on rs-FC using an ANCOVA model where rs-FC at rescan was predicted by intervention group with baseline rs-FC as a covariate (Vickers and Altman, 2001). The intervention group effect reflects the differential effect (ie, active $v s$ placebo) on change in rs-FC. This model was applied at a voxel-level in R v3.0.2 (http://cran.r-project.org/) as described previously (Fisher et al, 2014). Effects were visualized using SPM8, xjView (http://www.alivelearn.net/xjview) and Freesurfer (surfer. nmr.mgh.harvard.edu). We evaluated the association between change in depressive scores (score at rescan minus baseline) and rs-FC (estimate at rescan minus baseline) in only the participants who received $\mathrm{GnRHa}$ intervention to probe rs-FC related to individual variability in $\mathrm{GnRHa}$ responsiveness. Where significant effects were observed, mean rs-FC estimates within significant clusters were extracted for visualization. All locations are described in MNI coordinates.

We have previously reported that GnRHa significantly reduced estradiol levels relative to placebo and that the net decrease in estradiol predicted the emergence of depressive symptoms (Frokjaer et al, 2015). Therefore, where we observed a significant effect of GnRH intervention on rs-FC, we evaluated whether the GnRHa induced change in estradiol levels mediated that effect. In addition, where we observed an association between change in rs-FC and depressive symptoms, we extracted the corresponding cluster and evaluated the extent to which change in rs-FC-mediated the association between change in estradiol levels and change in depressive symptoms. Mediation effects were estimated using a structural equation model and approximate standard errors were estimated using the delta method. These confidence intervals were compared with non-parametric bootstrap estimates, which produced similar results. Unstandardized and standardized parameter estimates (b') are reported for mediation effects. Models were estimated using the lava package in R. $p$-values reflect two-tailed tests and $p<0.05$ was considered statistically significant.

\section{RESULTS}

\section{Hormone Profiles, and Depressive Symptoms Across Intervention Period}

As described previously, hormone responses across the study period confirmed the expected biphasic ovarian hormone response including an initial stimulation and a subsequent ovarian suppression to menopausal estradiol levels (Frokjaer et al, 2015). Similarly, GnRHa increased Hamilton scores from baseline $(p=0.003)$ and relative to placebo $(p=0.02)$ (Frokjaer et al, 2015). At a single-item level we observed that changes in Hamilton scores from baseline in the GnRHa group were distributed across symptom categories (eg, mood, energy level, sleep, anxiety, and somatic components).

\section{Baseline rs-FC and Main Effects of GnRHa Intervention}

At baseline we observed distributed regions showing statistically significant rs-FC with our seeds as expected (Figure 1). We did not observe evidence for a statistically significant main effect of GnRHa intervention on any rs-FC seed including the DMN as determined by our PCC seed.
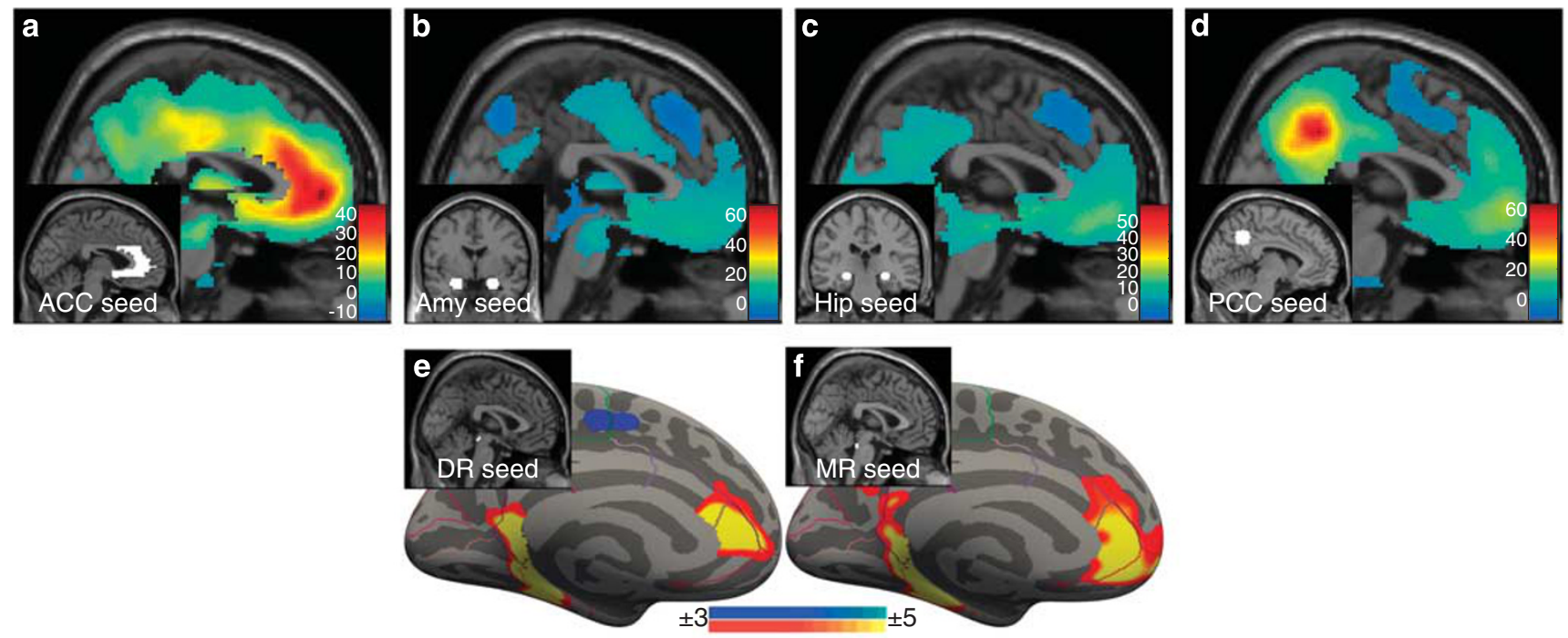

Figure I Resting-state functional connectivity maps across participants at baseline. (a-d) Statistical parametric maps showing areas significantly correlated with (a) anterior cingulate cortex, (b) amygdala, (c), hippocampus, (d) posterior cingulate cortex. Color bars represent t-scores. Sagittal slice is $X=0$. (e and f) Surface maps showing medial wall areas significantly correlated with (e) dorsal raphe and ( $f$ ) median raphe seeds as reported previously (Beliveau et al, 20 I 5). Color bars represent negative $\log _{10} p$-values. Warm colors denote a positive correlation whereas cool colors denote a negative correlation. Outline of respective seed inset. 

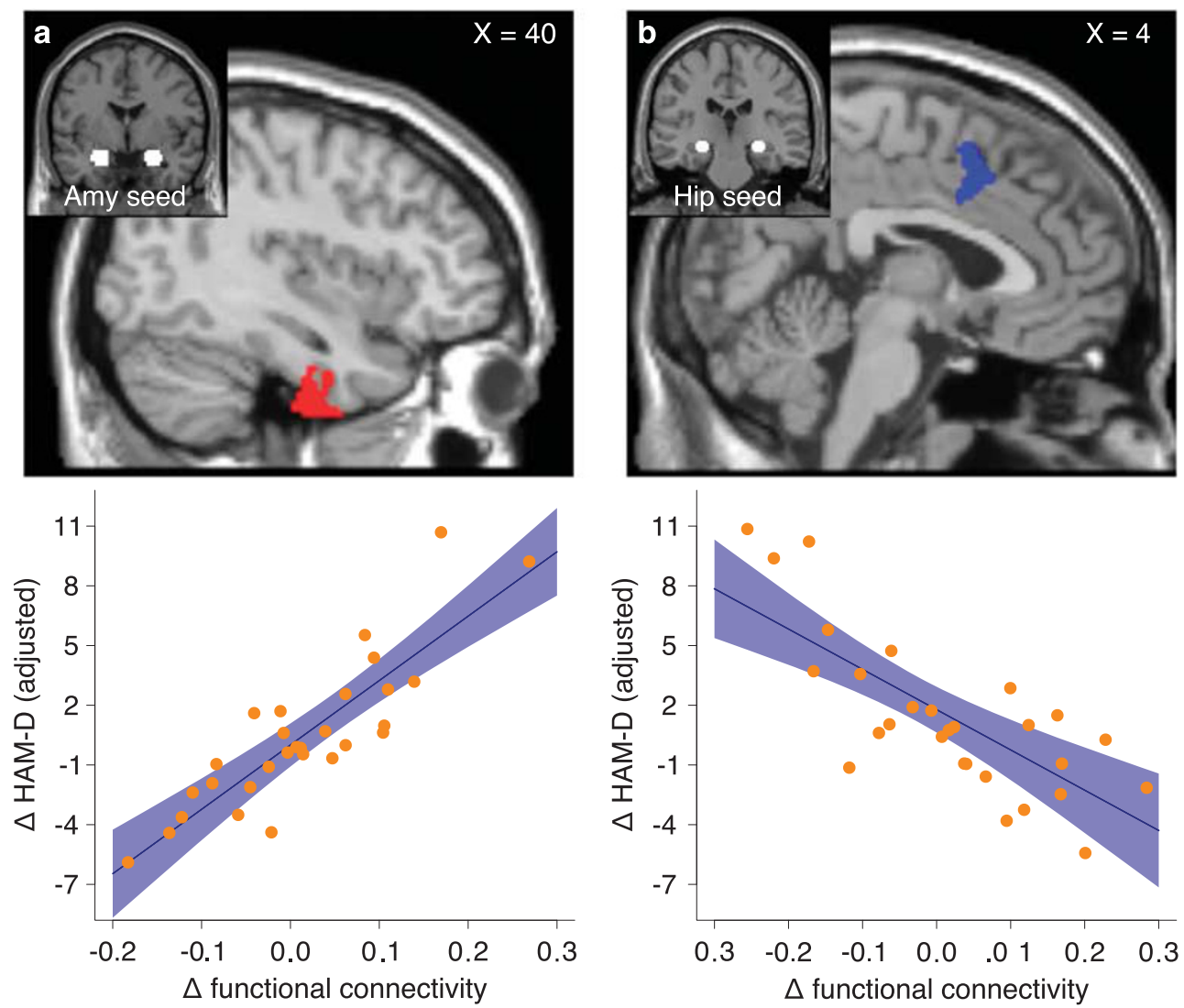

Figure 2 Emergence of depressive symptoms associated with change in rs-FC. Top, statistical parametric map showing (a) right temporal cortex, (b) cingulate gyrus/pre-SMA where change in rs-FC with respective seed (inset) was significantly associated with change in depressive symptoms. Red and blue denote a positive and negative correlation, respectively. Bottom, plot of mean change in respective functional connectivity estimates against change in depressive symptoms (change scores: rescan—baseline, adjusted for baseline depressive symptoms). Orange points represent individual mean functional connectivity estimates. Blue line and shading represents fit line and 95\% confidence limits on fit line, respectively. Amy, amygdala; Hip, hippocampus; $\Delta H A M-D$, change in Hamilton 17-item depression score.

\section{Change in Depressive Symptoms and Resting-State Functional Connectivity}

To identify connectivity measures associated with the individually variable response to GnRHa intervention, we determined the association between change in depressive symptoms and change in rs-FC in the group receiving GnRHa intervention $(n=29)$. A positive change score reflects an increase from baseline (ie, rescan minus baseline) for depressive symptoms and rs-FC.

For the amygdala seed we observed a cluster in right temporal cortex where change in depressive symptoms was positively associated with change in functional connectivity $(24,0,-50 ; z=3.90, k=331$ voxels, Figure $2 \mathrm{a})$. Conversely for the hippocampus seed, we observed a cluster encompassing cingulate gyrus and pre-supplementary motor area (preSMA) where change in depressive symptoms was negatively associated with change in hippocampus functional connectivity $(-4,16,48 ; z=4.33, k=211$ voxels, Figure $2 \mathrm{~b})$. There were no clusters wherein change in rs-FC with our dorsal raphe, median raphe, PCC, or ACC seeds was significantly associated with change in depressive symptoms.

Next we tested whether change in rs-FC in the above clusters mediated the negative association between net change in estradiol and change in depressive symptoms (ie, a larger decrease in estradiol levels predicted a larger increase in depressive symptoms; Figure 3). We observed effects wherein each cluster mediated the effect of change in estradiol on the emergence of depressive symptoms marginally outside our statistical significance threshold (mediation parameter estimate: amygdala-temporal cortex: -1.08 $(-2.26,0.11), \quad b^{\prime}-0.28, p=0.075$; hippocampus-ACC/preSMA: $-0.69(-1.75,0.36), b^{\prime}=-0.18, p=0.19$; Table 2). Although not statistically significant, these models indicate that a greater decrease in estradiol following GnRHa was associated with a larger increase in depressive symptoms via alterations in rs-FC.

\section{DISCUSSION}

Here we evaluated the effects of GnRHa intervention on rsFC with seed brain areas centrally involved in emotional behavior and previously associated with fluctuations in ovarian sex-steroid hormones. GnRHa intervention did not significantly affect rs-FC any of the six seeds we evaluated. We observed clusters where change in rs-FC with our amygdala and hippocampus seeds were correlated with change in depressive symptoms in the GnRHa group. These rs-FC measures partly mediated the association between change in estradiol and the emergence of depressive symptoms, providing novel evidence that substantial 


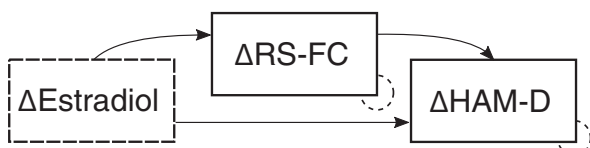

Figure 3 Schematic of mediation model to evaluate indirect effect of change in estradiol on change in depressive symptoms via change in rs-FC. Direct and mediation effects are described in Table 2. The mediation effect is the product of the two top arrows. The $\Delta$ estradiol $\rightarrow \Delta$ HAM-D connection is the direct effect. The total effect is the sum of the mediation and direct effects.

Table 2 Change in Estradiol Effects on Emergence of Depressive Symptoms Mediated by Change in Functional Connectivity ( $\mathrm{GnRHa}$ Group Only)

\begin{tabular}{llll}
\hline Connectivity/model path & Estimate & $\mathbf{9 5 \%} \mathbf{~ C l}$ & p-value \\
\hline Amygdala - right temporal cortex & & & \\
Total effects: direct+mediation effects & -1.40 & $(-2.79 ;-0.014)$ & 0.048 \\
Direct effects: $\Delta$ estradiol $\rightarrow \Delta$ HAMD & -0.32 & $(-1.11 ; 0.48)$ & 0.43 \\
Mediation effects: $\Delta$ estradiol $\rightarrow \Delta$ RS- & -1.08 & $(-2.27 ; 0.1$ I $)$ & 0.074 \\
FC $\rightarrow \Delta$ HAMD & & & \\
& & & \\
Hippocampus -cingulate gyrus/pre-SMA & & & \\
Total effects: direct+mediation effects & -1.67 & $(-3.07 ;-0.25)$ & 0.020 \\
Direct effects: $\Delta$ estradiol $\rightarrow \Delta$ HAMD & -0.98 & $(-1.96 ; 0.014)$ & 0.053 \\
Mediation effects: $\Delta$ estradiol $\rightarrow \Delta$ RS- & -0.69 & $(-1.75 ; 0.36)$ & 0.19 \\
FC - $\rightarrow \Delta$ HAMD & & & \\
\hline
\end{tabular}

Baseline RS-FC included as additional predictor of $\triangle \mathrm{HAMD}$.

fluctuations in sex-steroid hormones contribute to heightened risk for and emergence of depressive symptoms in part through effects on intrinsic functional connectivity.

Notably, GnRHa did not significantly modulate rs-FC in any seeds, which may reflect individual variability in GnRHa intervention sensitivity. This is conceptually consistent with the observation that only a subset of women who experience marked sex-steroid hormone fluctuations during, for example, pre- to post-partum or menopausal transition, develop depressive symptoms. Therefore, we considered whether individual variability in the emergence of depressive symptoms following GnRHa intervention mapped onto change in rs-FC. The emergence of depressive symptoms was significantly positively associated with rs-FC between the amygdala and right temporal cortex, whereas it was negatively correlated with rs-FC between hippocampus and a cluster including cingulate gyrus/pre-SMA. Furthermore, we observed that change in rs-FC partly mediated the association between change in estradiol and the emergence of depressive symptoms, implicating intrinsic connectivity as an important neurobiological mediator of ovarian sex-steroid hormone changes on depressive symptoms. The high expression of sex-steroid receptors in hippocampus and amygdala supports its potential sensitivity to sex hormone signaling (Barth et al, 2015). Our findings nominally indicate that a decline in estradiol levels increases hippocampuscingulate/pre-SMA rs-FC but decreases hippocampus-cingulate/pre-SMA rs-FC, which contributes to the emergence of depressive symptoms. Our finding that change in depressive symptoms was negatively associated with change in hippocampus-cingulate/pre-SMA rs-FC is consistent with a previous study that reported decreased hippocampuscingulate rs-FC in PPD women compared with postpartum healthy controls (Deligiannidis et al, 2013). Although our mediation effects were not statistically significant, we note that even such effects, given our relatively small sample size, provide intriguing support for a role for intrinsic hippocampus and amygdala connectivity as mediators of increased risk for the emergence of depressive symptoms following sex-steroid hormone fluctuations.

It is perhaps surprising that GnRHa and the emergence of depressive symptoms were not associated with amygdalaprefrontal rs-FC, given its critical role in processing emotionally salient stimuli. However, previous studies of depression, including PPD, did not report differences in amygdala-mPFC rs-FC relative to controls (Anand et al, 2005; Chase et al, 2014) but evidence for differences in amygdala reactivity and connectivity to tasks (Moses-Kolko et al, 2010; Silverman et al, 2011). Thus, our null observation coincides with literature indicating that depression-related amygdala-mPFC alterations are identifiable during emotionally salient tasks rather than in resting-state. In addition, despite convergent evidence linking sex hormones and serotonin signaling (Amin et al, 2006; Bethea et al, 2002, 2009; Frokjaer et al, 2010; Moses-Kolko et al, 2003), we did not observe an effect of $\mathrm{GnRHa}$ intervention or the emergence of depressive symptoms on rs-FC with our raphe seeds. Although this may partly reflect challenges in measuring fMRI signal in a small brainstem region (eg, pulsation artifacts), we note that we used a subject-specific multimodal approach to mitigate these effects. Thus, detection of GnRHa intervention effects on serotoninrelated intrinsic connectivity may require larger samples.

Notably, this model may capture the onset phase of depressive symptoms (Frokjaer et al, 2015), reflecting brain architecture differing from an established depressed state. In profiling single-item Hamilton responses, changes in the GnRHa group were distributed across symptom categories (Frokjaer et al, 2015). Notably, sleep disturbances were a prominent feature of the GnRHa response, which often represent an early indicator of depressive episodes (Nutt et al, 2008), highlighting the potential of our study to model depressive episode onset. Nevertheless, one might speculate if this reflected side-effect phenomena, irrelevant to depressive symptoms. However, GnRHa significantly affected item clusters comprising mood, anxiety, and sleep components of the Hamilton score relative to placebo (Frokjaer et al, 2015). Additional side-effects (eg, hot flashes and headaches) were significant or showed trends in the GnRHa group only well after the intervention (Frokjaer et al, 2015) and thus did not confound the time period wherein rs-FC and Hamilton scores were collected.

Another period of substantial sex hormone fluctuations in women is perimenopause. This period is also characterized by increased risk for depressive symptoms (Freeman et al, 2006). We are unaware of previous resting-state studies probing the neural pathways mediating these effects (Comasco et al, 2014) but note that our GnRHa protocol reflects a relevant model and our findings provide initial evidence for resting-state networks sensitive to perimenopausal-like fluctuations in sex- 
steroid hormones, which can serve as a benchmark for future studies evaluating such changes.

Our study is not without limitations. Limited withinsubject test-retest reliability is a challenge facing rs-fMRI studies (Franco et al, 2013) and we cannot rule out that this negatively affected our ability to identify GnRHa-induced changes in rs-FC. The study population was potentially biased toward low-to-average risk for developing even subclinical depressive symptoms because robust individuals may be more likely to participate in a large and demanding imaging program. This would lower our sensitivity to detect GnRHa effects relevant to more vulnerable populations. A broad aim of this study was to establish a pharmacological manipulation model that could be used to study sex hormone fluctuations in a controlled environmental setting. Nevertheless, future study of post-partum, perimenopausal women in a clinical setting or other relevant high-risk groups would further elucidate the relevance of our present results. We cannot disentangle separate ovarian steroid hormone effects because their fluctuations are coupled across phases. However, the model produced the largest contrast between baseline and follow-up estradiol levels, suggesting it is a particularly sensitive marker of intervention. Although we aimed to characterize the effect of a biphasic ovarian hormone manipulation, directly evaluating isolated phasespecific effects (eg, initial estradiol flare component) would have been interesting. However, this was not feasible owing to inherent uncertainty about the exact timing of the initial estradiol peak.

The analyses linking change in depressive symptoms and rs-FC are correlational and do not establish causality. Although the structural equation models assume directionality that is not necessarily established, we think that our model, wherein change in molecular signaling (estradiol) leads to brain network changes that affect behavior, is particularly sensible (Fisher and Hariri, 2012). Although highly relevant to delineating neurobiological pathways underlying risk for depression, our indirect effects were marginally significant within a small population for testing such effects. Therefore, we interpret them cautiously and acknowledge the need for future studies supporting these effects. It is possible that the emergence of depressive symptoms (or alterations in rs-FC) affects neurobiological mechanisms beyond the 4-week time interval of our protocol, which our study is not situated to identify. Hence, we cannot exclude that such time-frame differences explain discrepancies between our findings and studies including individuals with more fully established depressive states.

In summary, we present evidence that the emergence of depressive symptoms following $\mathrm{GnRHa}$ intervention was significantly associated with altered rs-FC with amygdala and hippocampus seeds. This rs-FC partly mediated the association between change in estradiol levels and the emergence of depressive symptoms, suggesting they may represent relevant neurobiological pathways through which fluctuations in sexsteroid hormones increase risk for depression. These findings provide novel insight into neurobiological mechanisms linking the emergence of depressive symptoms and heightened risk for depression with substantial alterations in sex hormone levels during critical phases of a woman's life.

\section{FUNDING AND DISCLOSURE}

VGF has received honorarium as speaker for $\mathrm{H}$ Lundbeck A/S. GMK has received honoraria as Field Editor of the International Journal of Neuropsychopharmacology and as scientific advisor for $\mathrm{H}$ Lundbeck A/S. HRS has received honoraria as Handling and Senior Editor for NeuroImage, as speaker for Biogen Idec Denmark A/S, and Scientific Advisor for Lundbeck A/S, Valby, Denmark, and has received financial travel support by Magventure, Skovlunde, Denmark. Trial Name: Neuropsychobiological Correlates of Sexsteroid Hormone Manipulation in Healthy Women: a Risk Model for Depression (GnRHa). Trial registration: www. clinicaltrials.gov. Trial ID: NCT02661789. All other authors declare no conflict of interest. The study was funded by The Danish Council for Independent Research, The Lundbeck Foundation (Cimbi), and The Capital Region of Denmark, Foundation for Health Research. SH was funded partly by the Swedish Research Council.

\section{ACKNOWLEDGMENTS}

Findings herein were presented as a poster entitled Resting State Functional Connectivity Signatures of Pharmacologically Induced Sex Hormone Fluctuations in a Risk Model for Depression at the 2015 Society of Biological Psychiatry meeting in Toronto, Canada. We wish to thank Elisabeth C. Larsen, Dorthe Givard, Agnete Dyssegaard, Anna Pors Nielsen, and Kenda Christensen for superb technical assistance.

\section{REFERENCES}

Amin Z, Gueorguieva R, Cappiello A, Czarkowski KA, Stiklus S, Anderson GM et al (2006). Estradiol and tryptophan depletion interact to modulate cognition in menopausal women. Neuropsychopharmacology 31: 2489-2497.

Anand A, Li Y, Wang Y, Wu J, Gao S, Bukhari L et al (2005). Activity and connectivity of brain mood regulating circuit in depression: a functional magnetic resonance study. Biol Psychiatry 57: 1079-1088.

Arelin K, Mueller K, Barth C, Rekkas PV, Kratzsch J, Burmann I et al (2015). Progesterone mediates brain functional connectivity changes during the menstrual cycle-a pilot resting state MRI study. Front Neurosci 9: 44.

Baker KG, Halliday GM, Tork I (1990). Cytoarchitecture of the human dorsal raphe nucleus. J Comp Neurol 301: 147-161.

Barth C, Villringer A, Sacher J (2015). Sex hormones affect neurotransmitters and shape the adult female brain during hormonal transition periods. Front Neurosci 9: 37.

Behzadi Y, Restom K, Liau J, Liu TT (2007). A component based noise correction method (CompCor) for BOLD and perfusion based fMRI. Neuroimage 37: 90-101.

Beliveau V, Svarer C, Frokjaer VG, Knudsen GM, Greve DN, Fisher PM (2015). Functional connectivity of the dorsal and median raphe nuclei at rest. Neuroimage 116: 187-195.

Bethea CL, Lu NZ, Gundlah C, Streicher JM (2002). Diverse actions of ovarian steroids in the serotonin neural system. Front Neuroendocrinol 23: 41-100.

Bethea CL, Reddy AP, Tokuyama Y, Henderson JA, Lima FB (2009). Protective actions of ovarian hormones in the serotonin system of macaques. Front Neuroendocrinol 30: 212-238.

Bloch M, Schmidt PJ, Danaceau M, Murphy J, Nieman L, Rubinow DR (2000). Effects of gonadal steroids in women with a history of postpartum depression. Am J Psychiatry 157: 924-930. 
Chase HW, Moses-Kolko EL, Zevallos C, Wisner KL, Phillips ML (2013). Disrupted posterior cingulate-amygdala connectivity in postpartum depressed women as measured with resting BOLD fMRI. Soc Cogn Affect Neurosci 9: 1069-1075.

Chase HW, Moses-Kolko EL, Zevallos C, Wisner KL, Phillips ML (2014). Disrupted posterior cingulate-amygdala connectivity in postpartum depressed women as measured with resting BOLD fMRI. Soc Cogn Affect Neurosci 9: 1069-1075.

Comasco E, Frokjaer VG, Sundstrom-Poromaa I (2014). Functional and molecular neuroimaging of menopause and hormone replacement therapy. Front Neurosci 8: 388.

Deligiannidis KM, Sikoglu EM, Shaffer SA, Frederick B, Svenson AE, Kopoyan A et al (2013). GABAergic neuroactive steroids and resting-state functional connectivity in postpartum depression: a preliminary study. J Psychiatr Res 47: 816-828.

Fisher PM, Hariri AR (2012). Linking variability in brain chemistry and circuit function through multimodal human neuroimaging. Genes Brain Behav 11: 633-642.

Fisher PM, Madsen MK, Mc Mahon B, Holst KK, Andersen SB, Laursen HR et al (2014). Three-week bright-light intervention has dose-related effects on threat-related corticolimbic reactivity and functional coupling. Biol Psychiatry 76: 332-339.

Fox MD, Snyder AZ, Vincent JL, Corbetta M, Van Essen DC, Raichle ME (2005). The human brain is intrinsically organized into dynamic, anticorrelated functional networks. Proc Natl Acad Sci USA 102: 9673-9678.

Franco AR, Mannell MV, Calhoun VD, Mayer AR (2013). Impact of analysis methods on the reproducibility and reliability of restingstate networks. Brain Connect 3: 363-374.

Freeman EW, Sammel MD, Boorman DW, Zhang R (2013). Longitudinal pattern of depressive symptoms around natural menopause. JAMA Psychiatry 71: 36-43.

Freeman EW, Sammel MD, Lin H, Nelson DB (2006). Associations of hormones and menopausal status with depressed mood in women with no history of depression. Arch Gen Psychiatry 63: 375-382.

Frokjaer VG, Erritzoe D, Juul A, Nielsen FA, Holst K, Svarer C et al (2010). Endogenous plasma estradiol in healthy men is positively correlated with cerebral cortical serotonin $2 \mathrm{~A}$ receptor binding. Psychoneuroendocrinology 35: 1311-1320.

Frokjaer VG, Pinborg A, Holst KK, Overgaard A, Henningsson S, Heede $M$ et al (2015). Role of serotonin transporter changes in depressive responses to sex-steroid hormone manipulation: a positron emission tomography study. Biol Psychiatry 78: 534-543.

Gould E, Tanapat P, Rydel T, Hastings N (2000). Regulation of hippocampal neurogenesis in adulthood. Biol Psychiatry 48: $715-720$.

Guintivano J, Arad M, Gould TD, Payne JL, Kaminsky ZA (2014). Antenatal prediction of postpartum depression with blood DNA methylation biomarkers. Mol Psychiatry 19: 633.

Henningsson S, Madsen KH, Pinborg A, Heede M, Knudsen GM, Siebner HR et al (2015). Role of emotional processing in depressive responses to sex-hormone manipulation: a pharmacological fMRI study. Transl Psychiatry 5: e688.

Hjelmervik H, Hausmann M, Osnes B, Westerhausen R, Specht K (2014). Resting states are resting traits-an FMRI study of sex differences and menstrual cycle effects in resting state cognitive control networks. PLoS One 9: e103492.

Kessler RC, McGonagle KA, Swartz M, Blazer DG, Nelson CB (1993). Sex and depression in the National Comorbidity Survey. I: lifetime prevalence, chronicity and recurrence. J Affect Disord 29: $85-96$.

Kranz GS, Hahn A, Savli M, Lanzenberger R (2012). Challenges in the differentiation of midbrain raphe nuclei in neuroimaging research. Proc Natl Acad Sci USA 109: E2000.

Lethaby A, Hogervorst E, Richards M, Yesufu A, Yaffe K (2008). Hormone replacement therapy for cognitive function in postmenopausal women. Cochrane Database Syst Rev 23: CD003122.

Lord C, Buss C, Lupien SJ, Pruessner JC (2008). Hippocampal volumes are larger in postmenopausal women using estrogen therapy compared to past users, never users and men: a possible window of opportunity effect. Neurobiol Aging 29: 95-101.

Macoveanu J, Henningsson S, Pinborg A, Jensen P, Knudsen GM, Frokjaer VG et al (2016). Sex-steroid hormone manipulation reduces brain response to reward. Neuropsychopharmacology 41: 1057-1065.

Mehta D, Newport DJ, Frishman G, Kraus L, Rex-Haffner M, Ritchie JC et al (2014). Early predictive biomarkers for postpartum depression point to a role for estrogen receptor signaling. Psychol Med 44: 1-14.

Mehta D, Quast C, Fasching PA, Seifert A, Voigt F, Beckmann MW et al (2012). The 5-HTTLPR polymorphism modulates the influence on environmental stressors on peripartum depression symptoms. J Affect Disord 136: 1192-1197.

Meltzer-Brody S, Boschloo L, Jones I, Sullivan PF, Penninx BW (2013). The EPDS-Lifetime: assessment of lifetime prevalence and risk factors for perinatal depression in a large cohort of depressed women. Arch Womens Ment Health 16: 465-473.

Moses-Kolko EL, Berga SL, Greer PJ, Smith G, Cidis Meltzer C, Drevets WC (2003). Widespread increases of cortical serotonin type $2 \mathrm{~A}$ receptor availability after hormone therapy in euthymic postmenopausal women. Fertil Steril 80: 554-559.

Moses-Kolko EL, Perlman SB, Wisner KL, James J, Saul AT, Phillips ML (2010). Abnormally reduced dorsomedial prefrontal cortical activity and effective connectivity with amygdala in response to negative emotional faces in postpartum depression. Am J Psychiatry 167: 1373-1380.

Munk-Olsen T, Laursen TM, Pedersen CB, Mors O, Mortensen PB (2006). New parents and mental disorders: a population-based register study. JAMA 296: 2582-2589.

Nutt D, Wilson S, Paterson L (2008). Sleep disorders as core symptoms of depression. Dialogues Clin Neurosci 10: 329-336.

Petersen N, Kilpatrick LA, Goharzad A, Cahill L (2014). Oral contraceptive pill use and menstrual cycle phase are associated with altered resting state functional connectivity. Neuroimage $\mathbf{9 0}$ 24-32.

Protopopescu X, Butler T, Pan H, Root J, Altemus M, Polanecsky M et al (2008). Hippocampal structural changes across the menstrual cycle. Hippocampus 18: 985-988.

Sanjuan J, Martin-Santos R, Garcia-Esteve L, Carot JM, Guillamat R, Gutierrez-Zotes A et al (2008). Mood changes after delivery: role of the serotonin transporter gene. $\mathrm{Br} J$ Psychiatry 193: 383-388.

Sellers K, Raval P, Srivastava DP (2015). Molecular signature of rapid estrogen regulation of synaptic connectivity and cognition. Front Neuroendocrinol 36: 72-89.

Silverman ME, Loudon H, Liu X, Mauro C, Leiter G, Goldstein MA (2011). The neural processing of negative emotion postpartum: a preliminary study of amygdala function in postpartum depression. Arch Womens Ment Health 14: 355-359.

Vickers AJ, Altman DG (2001). Statistics notes: Analysing controlled trials with baseline and follow up measurements. BMJ 323: 1123-1124.

Whitfield-Gabrieli S, Nieto-Castanon A (2012). Conn: a functional connectivity toolbox for correlated and anticorrelated brain networks. Brain Connect 2: 125-141.

Woolley CS, McEwen BS (1992). Estradiol mediates fluctuation in hippocampal synapse density during the estrous cycle in the adult rat. J Neurosci 12: 2549-2554.

Yankova M, Hart SA, Woolley CS (2001). Estrogen increases synaptic connectivity between single presynaptic inputs and multiple postsynaptic CA1 pyramidal cells: a serial electronmicroscopic study. Proc Natl Acad Sci USA 98: 3525-3530. 